\title{
ANALYSIS OF AIRCRAFT CONFLICT GEOMETRIES IN EUROPE
}

\author{
Rudi Ehrmanntraut, EUROCONTROL Experimental Centre (EEC) \\ Raphaël Christien, Pacte Novation, Brétigny sur Orge, France
}

\begin{abstract}
Conflict Management is a central role of air traffic control. Several projects in the world work on the semi- or full-automation of this function. The understanding of the nature of aircraft conflict is a prerequisite for successful automation. Therefore, it would be useful to update the statistics about aircraft conflicts in Europe, to know where and when they occur, the distribution of attitudes of involved aircraft, of encounter angles, encounter speeds, encounter flight levels, and flight phases at the time of conflict. Of special interest are also statistics about proximities and correlations with other parameters of airspace complexity.

The results of the European-wide simulation give new insights into the nature of conflicts. The studies have been conducted with the help of a simulation model. The simulation tool is presented, and the assumptions and conditions of the simulations described. The empirical data is used for a theoretical discussion about uncertainty of conflict prediction.
\end{abstract}

\section{Introduction}

The mission of Air Traffic Management (ATM) is the safe, orderly and expeditious management of air traffic. One of its key functions is Conflict Management [1], i.e. the detection of air traffic conflicts and their resolution. Today air traffic controllers execute this task. It is expected that the semi- or even full automation of this function would give benefits by increasing capacity and safety, could lead to more economical flights, and eventually cut ATM operations costs. A number of projects on both sides of the Atlantic ${ }^{12}$ work on the issue, which have in common the application of the human-centred paradigm and a

\footnotetext{
${ }^{1}$ U.S.A. : URET (http://www.caasd.org/proj/uret/), CTAS (http://www.ctas.arc.nasa.gov/)

${ }^{2}$ Europe : ASA

(http://www.eurocontrol.int/eatmp/work/auto supp ats.html), ERATO (http://www.tls.cena.fr/divisions/CEP/ERATO/)
}

step-wise implementation starting with mediumterm conflict detection and slowly introducing decision aids for conflict resolution. Steps towards full-automation are pure research at the moment.

The understanding of the nature of aircraft conflict is a prerequisite for successful automation. There is relatively little previous work on empirical measures. Magill [3] is possibly the most cited document, analysing conflict geometries and interpreting trajectory uncertainties. Granger [4] has focussed on clusters of conflicts and possible conflicts depending on uncertainty of predicted trajectories and is limited to simulations with French traffic. Cloerec [5] gives statistics about conflict encounter geometries; simple clusters with two aircraft in conflict against more complex clusters; and simple proximities. The study is limited to the Maastricht en-route airspace.

This works tries to update and refine some basic figures using a model simulator running $\mathrm{ECAC}^{3}$ traffic.

\section{The Simulator}

The COLA (Complexity Light Analyser) complexity simulator was used for this study, which is developed at EEC. It treats environment data such as routes, sectors, navaids, airports, military areas, CFMU traffic volume descriptions; flight plan data, control centres configurations, capacity of sectors/collapsed sectors/traffic volumes, opening schemes; flow regulation schemes, and CFMU regulation plans as input data.

The produced outputs are basic information such as number of flight plans, conflict information, sector information, more granular information on simulator internal cubes, statistical indicators on complexity factors such as instantaneous number of aircraft, number of times the number of entering aircraft exceeded a defined threshold.

\footnotetext{
${ }^{3}$ European Civil Aviation Conference
} 


\section{Simulated Traffic and Conflict Model}

The simulation traffic covers one entire day June, 132003 for all ECAC. The computed trajectories are extracted from the CFMU ${ }^{4}$ and corrected with $\mathrm{ETFMS}^{5}$ data, which corresponds to the real executed trajectories. The correction only takes place when the radar data deviates by more than $20 \mathrm{NM}$ or 5 minutes lateral or 700 feet vertical. $4 \mathrm{D}$ trajectories are simple in COLA.

The used conflict model was a block of $5 * 5 \mathrm{NM}$ lateral and 1000 feet horizontal dimensions. If an aircraft pair has several conflicts, it is counted the first time and only once, assuming that in reality conflicts would be resolved once and for ever.

\section{Results and Interpretation}

\section{Encounter Angles and Attitudes}

The distribution of attitudes for all flights above flight level (FL) 60 is shown in Figure 1 and all flights above FL 180 in Figure 2. The cut at FL 180 splits the conflicts into almost two equal parts, e.g. $50 \%$ of conflicts occur above and $50 \%$ below FL 180 .

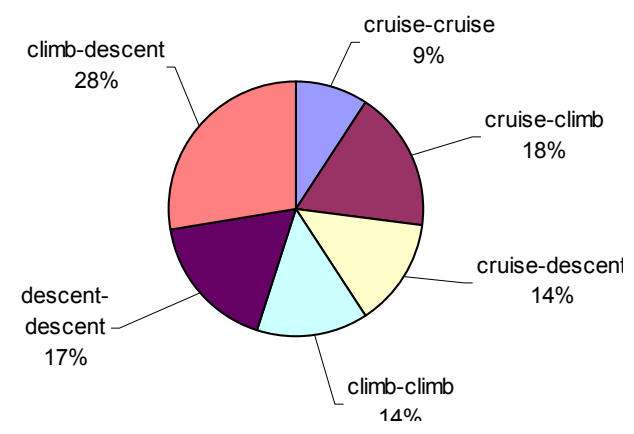

Figure 1. Conflict Attitudes above FL60

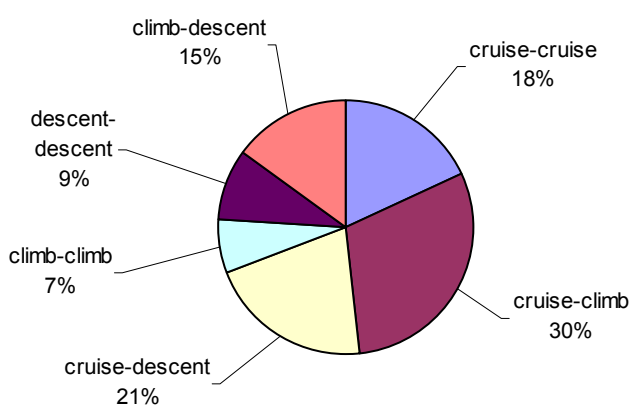

Figure 2. Conflict Attitudes above FL180

It can be reconfirmed that only a small percentage of conflicts are cruise-cruise encounters. At least one aircraft is in cruise for $41 \%$ below and $69 \%$ above FL 180. At least one aircraft is in climb for $60 \%$ below and $52 \%$ above FL 180 . At least one aircraft is in descent for $59 \%$ below and $45 \%$ above FL 180.

With this result it could be argued that most of the mathematical literature (e.g. [6]) on conflict resolution is too much simplifying by considering mainly the cruise-cruise encounters, which are only a relatively small part of the problem even in the en-route environment.

Figure 3 shows on a logarithmic scale the sum of all conflicts over the encounter angles and then refines into the categories cruise-cruise; cruiseclimb; cruise-descent; climb-climb; climb-descent; and descent-descent. It can be seen that there are very strong peaks at $0^{\circ}$ and $180^{\circ}$ with $22 \%$ and $9 \%$ of all conflicts respectively. Between these extremes the distribution is almost equal but differs for the different attitudes of the aircraft in conflict.

Figure 4 shows the same values filtered from the $0^{\circ}$ and $180^{\circ}$ on a linear scale to distinguish the attitudes. Climb-descent strongly increases with the large encounter angles. Climb-climb decreases with the first $45^{\circ}$; and together all attitude-attitude conflicts have a minimum at $70^{\circ}$. Cruise-attitude conflicts increase for angles smaller than $25^{\circ}$ and bigger than $140^{\circ}$; and cruise-cruise have the inverse behavior.

\footnotetext{
${ }^{4}$ EUROCONTROL Central Flow Management Unit

${ }^{5}$ Enhanced Tactical Flow Management System, surveillance picture over ECAC with 3 minutes update rates.
} 


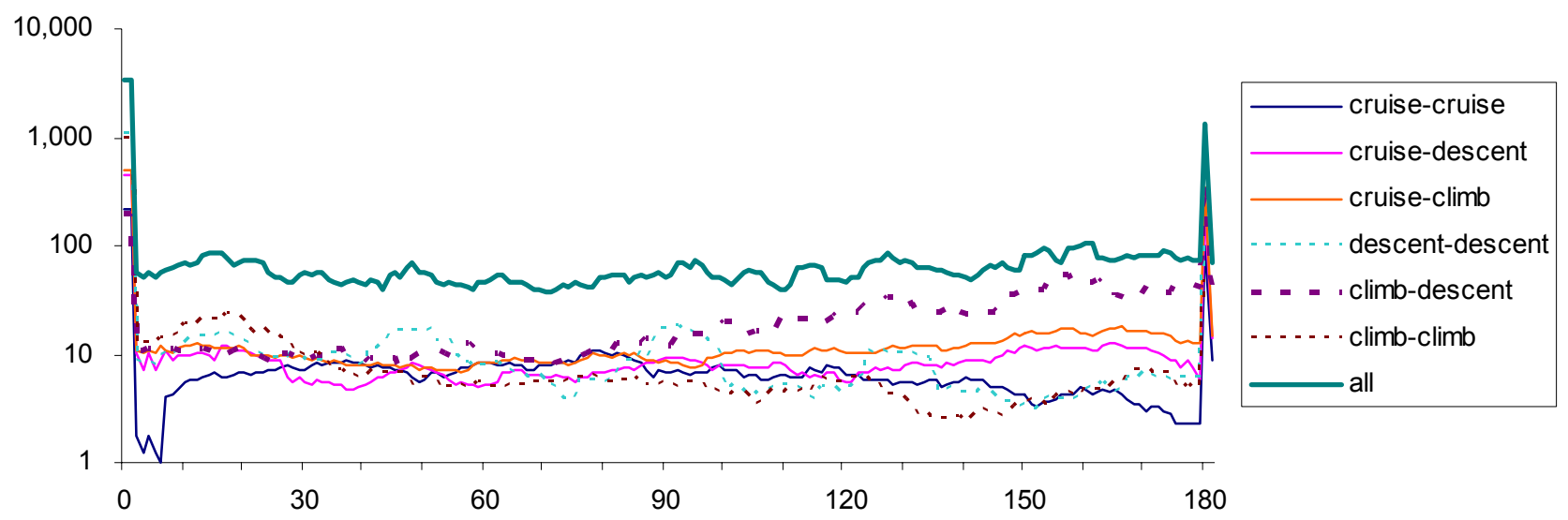

Figure 3. Number (Log) of Conflicts over Encounter Angles by Attitude

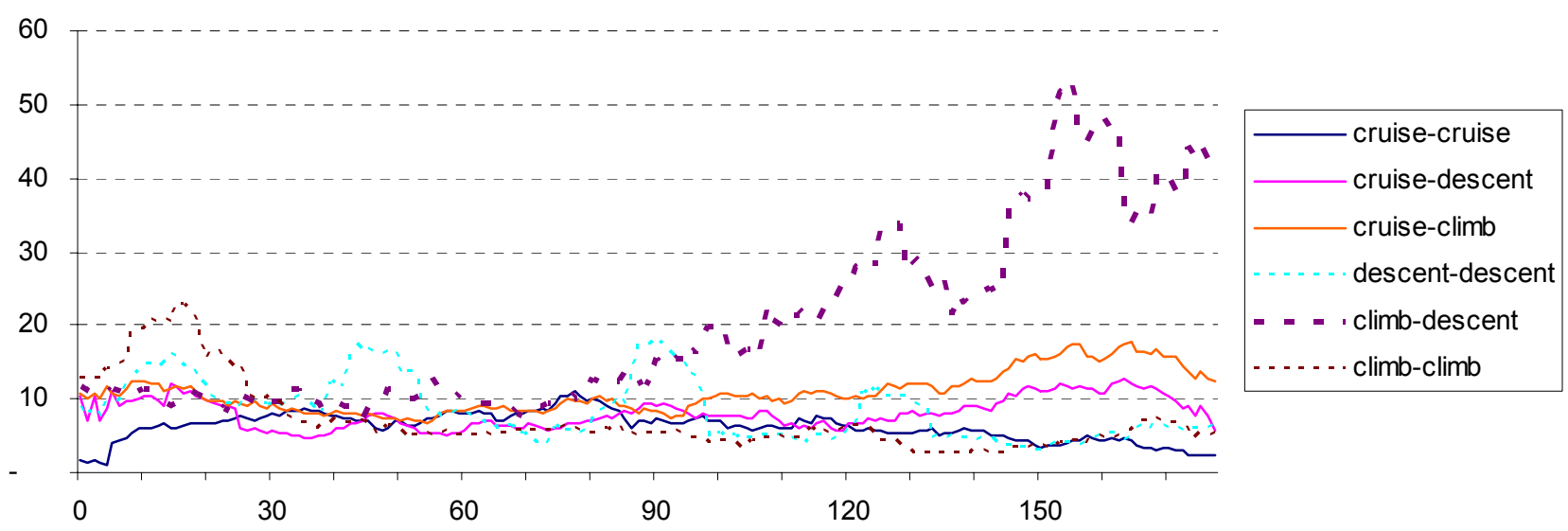

Figure 4. Number of Conflicts over Encounter Angles by Attitude except $0^{\circ}$ and $180^{\circ}$

The interpretation is that:

- Overtaking or sequencing is the reason for about $30 \%$ of all conflicts.

- Next highest category is climb-descent, which occurs most often around airports.

- All other categories are almost equally distributed.

Figure 5 groups all the angles into three main categories: in-trail, cross and opposite, where the opening angles are $\pm 15^{\circ}$ and $\pm 165^{\circ}$. This corresponds to usual differentiation done by air traffic controllers.

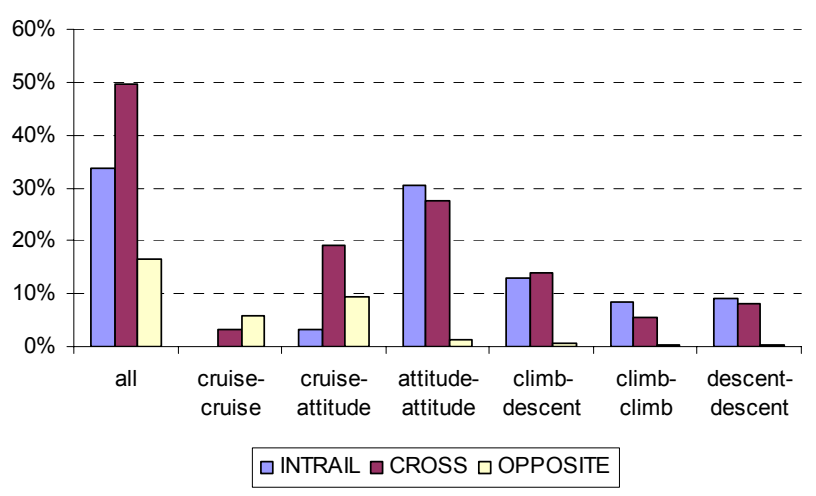

Figure 5. Percentage of Conflicts over Encounter Angle Category by Attitude 


\section{Encounter Flight Levels}

The following graphs show the percentage of conflicts per flight level band and per angle category. The legend can be found in the Figure 12 .

Figure 6 shows the conflict distributions for all attitudes per FL band. One FL band was arbitrarily defined as 5 flight levels for easier visualization. There is a peak for the low FLs and for FL 310 to 350 , the typical cruise altitudes. In-trail and opposite together are higher than crossing conflicts for all FL bands. From FL 160 to FL 250 in-trail conflicts have the highest rating.

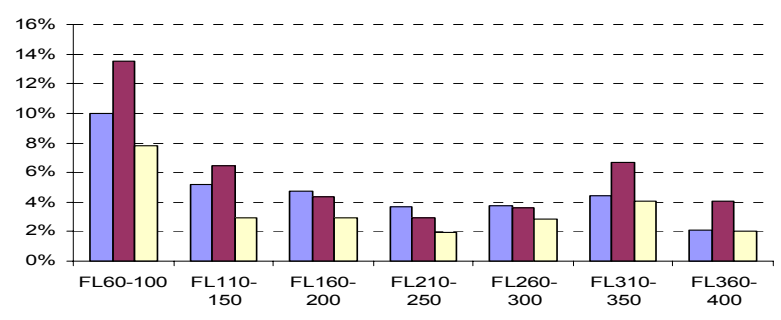

Figure 6. All Conflicts per FL Band and Angle Category

This is broken down into the different attitude groups in Figure 7, Figure 8, Figure 9, Figure 10, Figure 11 and Figure 12. The percentage is relative to the total number of conflicts.

It can be clearly seen that the conflicts with aircraft in climb or descent occur at the lower flight levels. When both aircraft are in cruise, climb or descent then there a very little opposite conflicts through all flight levels.

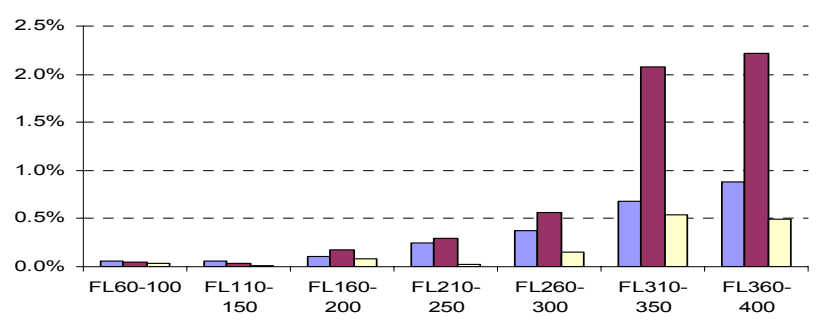

Figure 7. Both Aircraft in Cruise

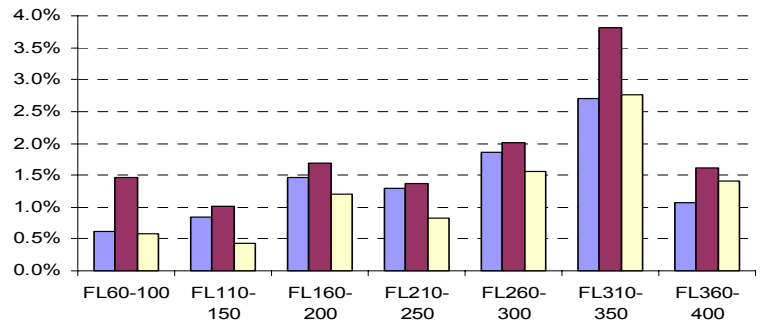

Figure 8. One Aircraft in Cruise, one in Climb OR Descent

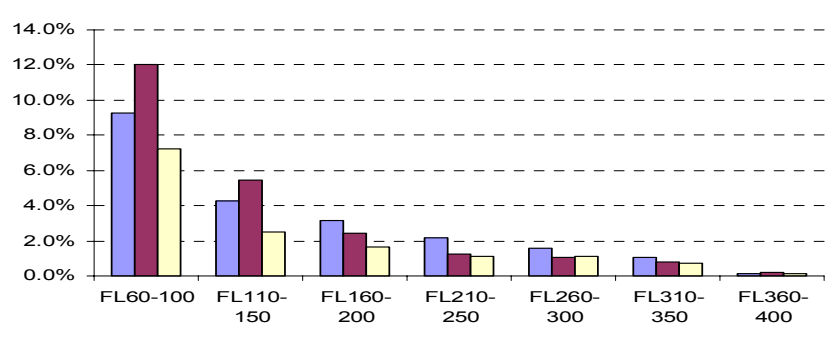

Figure 9. Both Aircraft in Climb OR Descent

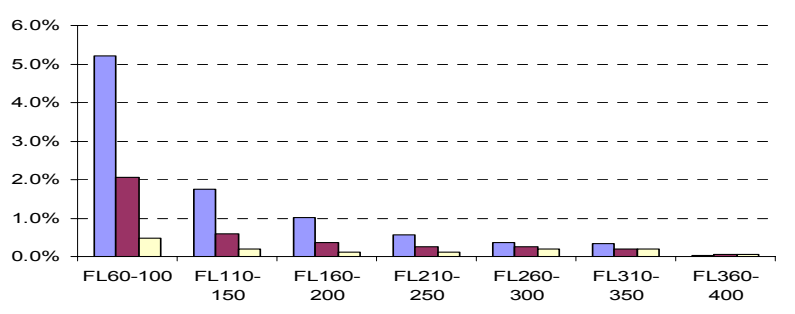

Figure 10. Both Aircraft in Climb

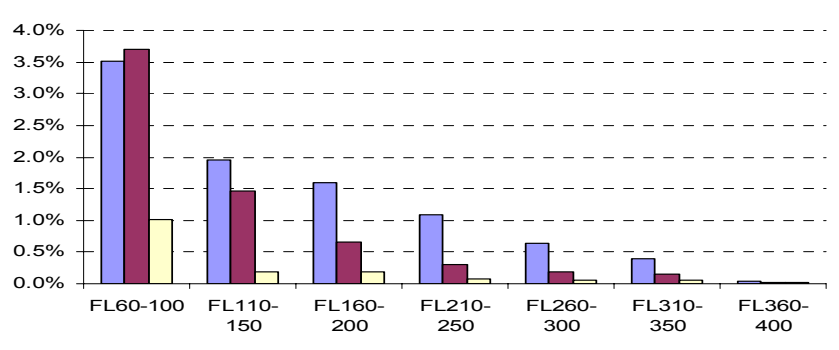

Figure 11. Both Aircraft in Descent 


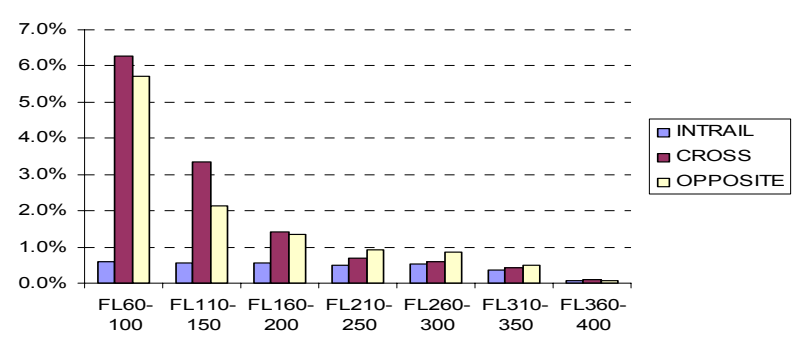

Figure 12. One Aircraft in Climb, the Other in Descent

\section{Encounter Speeds}

Figure 13 shows the quotient of encounter speeds. The anomalies are due to the retrieval of speeds, however parallel studies [7] with different tools confirm at least the asymptotical behavior towards the value of 1.1 for high altitudes. Conflicts at lower altitudes have higher speed differences towards a ratio of 2. Encounter angles are of no significance to the quotient of speeds. It can be derived that aircraft in evolution and in the lower flight levels have higher variances in speed. The speed quotient is important for the discussion of uncertainties.

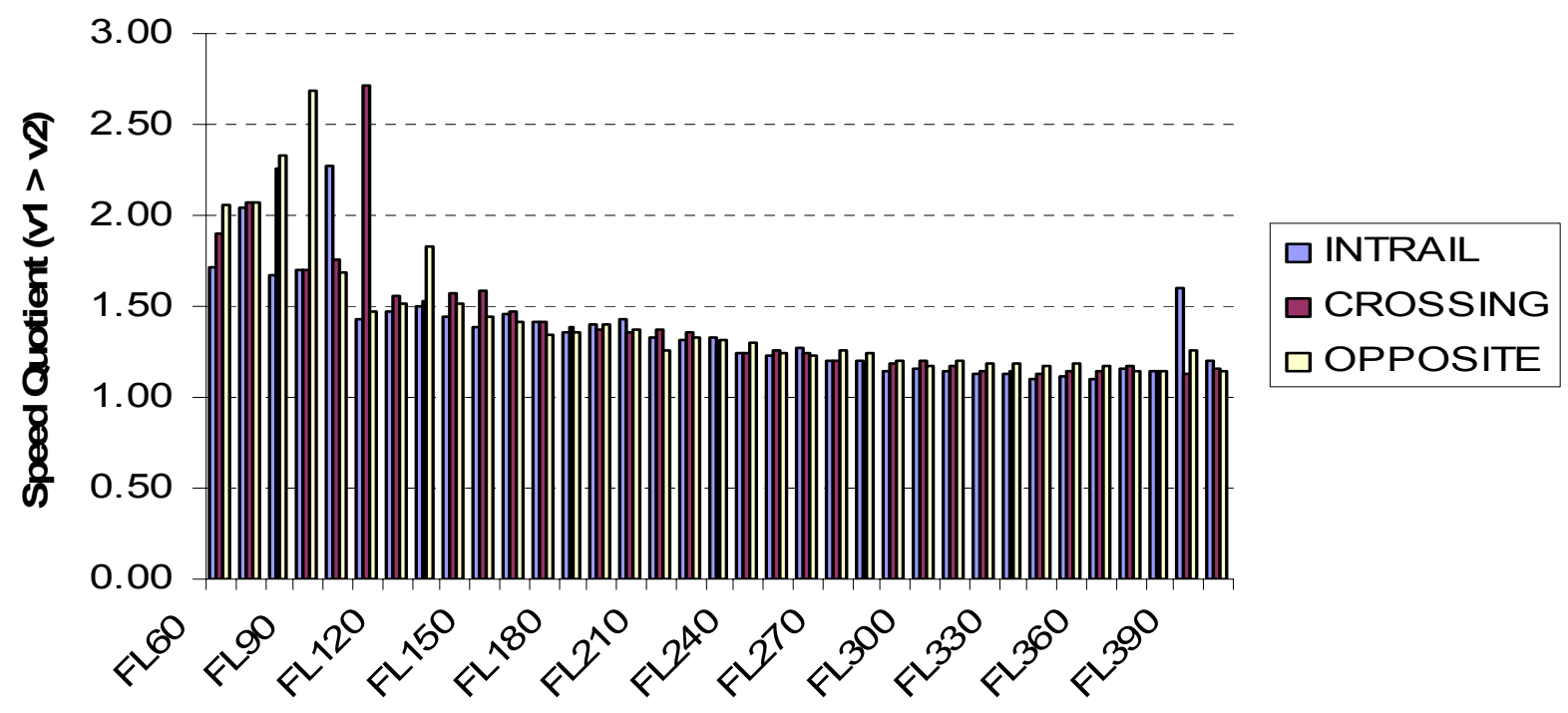

Figure 13. Speed Quotient per Flight Level and Angle Category

\section{Complexities}

One of the many parameters for complexity is the number of environmental aircraft around conflicts [8-10].

Figure 14 illustrates the average number of environmental aircraft at the same flight level as a function of the range for some control centers with very high conflict rates. In the simulated traffic sample there is in average one other aircraft in a range of $10 \mathrm{NM}$, about 5 within $25 \mathrm{NM}, 7$ within 40NM and 9 within 70NM.

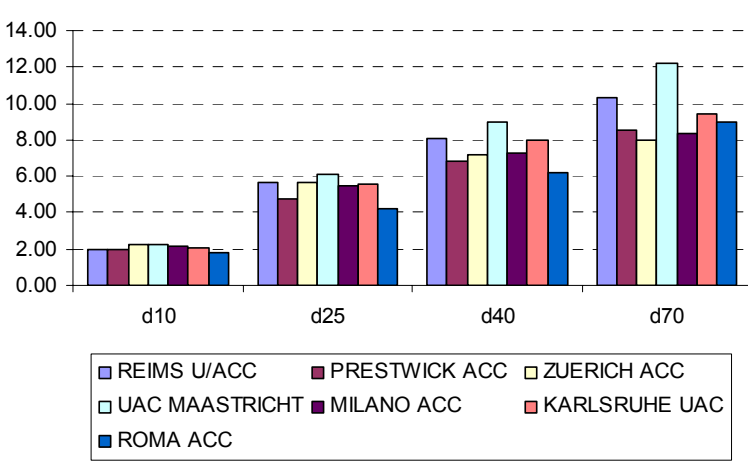

Figure 14. Average Number of Proximities in Radius of 10NM, 25NM, 40NM and 70NM 


\section{Discussion}

This discussion will focus on an interpretation of encounter speeds and angles when considering uncertainty of the prediction of conflict due to along track trajectory prediction errors. The mathematics are used as defined in Niedringhaus [10] and especially Irvine $[12,13]$.

The reliability of the prediction of the conflicts, their geographical location and time of conflict are of highest importance for all decision support tools. Uncertainty can lead to predicted conflicts that will finally not occur and herewith unnecessarily create workload. The higher the uncertainty is the more airspace buffers must be managed around the conflict and its resolution, which consumes the precious resource of airspace.

Irvine [13] finds a relation of encounter angle $\theta$ and quotient of speeds $m$ to describe the variance of the minimal displacement, expressed by a factor $\gamma^{2}$.

$$
\gamma^{2}=\frac{\left(\frac{1}{m}+m\right) \sin ^{2} \theta}{\left(\frac{1}{m}-2 \cos \theta+m\right)}
$$

An operational interpretation of this displacement variance would be that the higher this factor is, the higher the uncertainty of the predicted conflict is and the more buffer airspace is needed. Figure 15 shows the graph as a function of speed quotient over the encounter angles.

The interpretation based on the empirical values is as follows: Most conflicts are overtaking and opposite at $0^{\circ}$ and $180^{\circ}$ and it can be seen that they have very low variance, i.e. uncertainty is low and prediction of conflict good. However, all other conflicts are more or less equally distributed over all angles and no zone in the graph is more significant. The en-route ratios of speed are between 1.4 and 1.1 where the curve with the high variances applies with a maximum of 1.8 at about $20^{\circ}$. For the lower flight levels the ratio of speed is between 2 and 1.4 where a lower curve applies with a maximum of about 1.4 at about $60^{\circ}$.

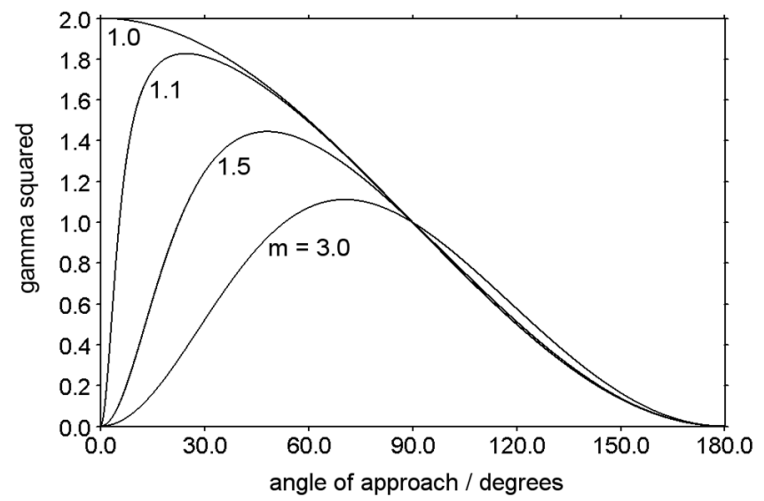

Figure 15. Minimum Displacement Variance against Angle of Approach for Various Ratios of Speed

\section{Conclusion}

This simulation analysed aircraft conflict geometries, the speeds and attitudes of conflicting aircraft at conflict flight levels with a simulation of one day traffic for the entire ECAC airspace. The simulation results presented in this paper confirm the previous smaller-scale studies by Magill [3] and Cloerec [5].

Only a small percentage of conflicts occur when both aircraft are cruising, 9\% below and 18\% above FL 180. That means that many of the mathematical models that have been created on conflict detection and resolution only treat a small and possibly the simplest part of the problem.

The distribution of encounter angles and speeds differ depending on attitudes and flight levels: low flight levels have many in-trail conflicts with high speed variances, whereas conflicts at higher altitudes are more equally distributed over angle and converge to a speed-ratio of 1.1.

The empirical results feed a discussion on the uncertainty of conflict prediction due to uncertainty of along track trajectory prediction. It found that many of the conflicts would not suffer from high variances in the prediction of along track errors and would therefore have low uncertainties, due to the very small and very large encounter angles. All other conflicts, however, would equally spread over all angles and not allow for further simplifications. The knowledge of the ratios of speed could be useful for system design to improve the conflict 
prediction tools as a function of their application, i.e. en-route will have to treat higher uncertainties than the TMA.

The study presents a step towards the analysis of complexity, where conflicts play a major role. Future empirical work will focus on clusters of conflicts and environmental aircraft, i.e. those other aircraft that are close to a conflict.

\section{Acknowledgements}

The authors would like to thank Richard Irvine for support and review, and Geraldine Flynn together with the COCA project team for the provision of resources.

\section{References}

[1] ICAO, Air Traffic Management Operational Concept Panel (ATMCP), 18 to 28 March 2002, first meeting, Montreal, Operational Concept Document

[2] Kauppinen, S., 2000, CORA1 Current Status Review, EUROCONTROL, ASA.01.CORA.1.DEL01.RPT

[3] Magill, S.A.N., Trajectory Predictability And Frequency Of Conflict-Avoiding Action, 1996, DERA (Quinetic)

[4] Granger, G., 2002, Détection et résolution de conflits aériens : modélisations et analyse

[5] Cloerec, A., K. Zeghal, E. Hoffman, 1999, Traffic Complexity Analysis To Evaluate The Potential For Limited Delegation Of Separation
Assurance To The Cockpit, in proceedings of the 18th DASC

[6] EUROCONTROL, 2002, Towards a controllerbased conflict resolution tool - a literature review

[7] Ehrmanntraut, R., 2004, The Potential Of Speed Control, EUROCONTROL Experimental Centre, in proceedings of the $23^{\text {rd }}$ DASC

[8] Chatterji, G., and B. Sridhar, 2001, Measures for Air Traffic Controller Workload Prediction. First AIAA Aircraft Technology, Integration, and Operations Forum, Los Angeles, CA.

[9] Laudeman, I., S. Shelden, R. Branstrom, and C. Brasil, 1998, Dynamic Density: An Air Traffic Management Metric. Report No. NASA/TM -1998112226. Moffett Field, CA: NASA.

[10] Kopardekar, P., 2000, Dynamic Density, A Review of Proposed Variables. Egg Harbor Twp., NJ: FAA NAS Advanced Concepts Branch, ACT540.

[11] Niedringhaus, W.P., Mathematical Formulation for Planning Automated Aircraft Separations for AERA 3, DOT/FAA/DS89/20, Appendix G, 1989, MITRE

[12] Irvine, R., 2001, A Geometrical Approach To Conflict Probability Estimation, EUROCONTROL Experimental Centre, in proceedings of the $4^{\text {th }}$ FAA/EUROCONTROL R\&D Seminar

[13] Irvine, R., 2003, Target Miss Distance To Achieve A Required Probability Of Conflict, EUROCONTROL Experimental Centre, in proceedings of the $5^{\text {th }}$ FAA/EUROCONTROL R\&D Seminar, Budapest 